\title{
Autoradiographic study of the permeability characteristics of the small intestine
}

\author{
J. G. C. KINGHAM,1 JUNE H. BAKER, AND C. A. LOEHRY \\ From the Department of Medicine, Royal Victoria Hospital, Bournemouth
}

SUMMARY This autoradiographic study demonstrates the distribution of a range of small solutes and macromolecules in the mucosa of the guinea-pig small intestine after intracardiac injection. The substances investigated were: ${ }^{14} \mathrm{C}$-urea, ${ }^{3} \mathrm{H}$-mannose, ${ }^{3} \mathrm{H}$-inulin, and ${ }^{125} \mathrm{I}$ polyvinylpyrrolidone (PVP). Small bowel biopsies were taken at intervals from one to 60 minutes after injection and the tissues processed for autoradiography. Light microscopic examination of the autoradiographs showed that the compartmental distribution depended on the molecular size of the substances being studied. Urea and mannose, as small solutes, were uniformly distributed throughout the intravascular, extravascular, and epithelial compartments. Inulin was evenly distributed in the vessel lumen and extravascular space but there was a considerable drop in concentration in the epithelium. PVP exhibited the most marked gradients, the concentration being greatest in the vascular lumina, lower in the extravascular space, least in the epithelium. Thus there appear to be two barriers to macromolecular passage which are freely permeable to small solutes: the capillary wall and the epithelium. At a light microscopical level it is not possible to observe whether the limiting membrane of each of these barriers is the cell plasmalemmal membrane or the basement membrane. The selectivity of the epithelial barrier is greater than that of the capillary barrier.

It is widely appreciated that the small intestine represents a semi-permeable barrier between the vascular lumen and the external environment of the intestinal lumen. This is a complex multiple barrier whose anatomical components comprise the vascular endothelium, the vascular basement membrane, the epithelial basement membrane, and the epithelium of the intestinal mucosa. The small intestine is a relatively impermeable barrier to the solutes whose molecular weight is greater than 180 (Fordtran et al., 1965), but it is well recognised that substances greatly in excess of this size may permeate the mucosa in minor degrees. Plasma proteins up to the size of IgM immunoglobulins certainly pass from the circulation into the intestinal lumen (Barth et al., 1964; Jarnum and Jensen, 1972) and substances as large as ferritin (Bockman and Winborn, 1966) and IgG (Kraehenbuhl and Campiche, 1969) are absorbed from the small intestine by passive sorption. Loehry

${ }^{1}$ Present address and address for communications: Dr J. G. C. Kingham, Department of Gastroenterology, St Bartholomew's Hospital, London EC1A 7BE.

Received for publication 9 August 1977 et al. (1970) have shown that intestinal permeability to any water solute, in both absorptive and exsorptive directions, is inversely dependent on the molecular weight of that solute. Kingham and Loehry (1976) and Kingham et al. (1976) using a perfusion technique have shown by selective damage to certain mucosal structure that the various anatomical barriers have different permeability functions. Thus the capillary endothelium was shown to be moderately selective but only mildly rate-limiting, the mucosal epithelium to be markedly rate-limiting but nonselective, and the epithelial basement membrane to be highly selective but minimally rate-limiting.

None of these studies, however, has given any direct morphological evidence of the mode or site of loss of the water solutes and macromolecules from the intestinal mucosa.

Autoradiography is a particularly useful technique in the investigation of dynamic mechanisms and it was thought that the technique could be successfully used to study the distribution of passively permeating solutes within a tissue. In view of the free diffusibility of water soluble substances within the tissues under study, it was not possible to use conventional tissue processing and autoradiographic techniques. Instead, 
theitissues were fixed by freezing and all subsequent autoradiographic procedures were performed in the frozen state; after exposure and development the sections could be stained in a routine manner.

The aims of the present study were to elucidate the permeability characteristics of the individual anatomical barriers of the small intestine by autoradiographic demonstration of the compartmental distribution of four widely varying molecular sized substances. Those chosen were urea (M.W. 60), mannose (M.W. 180), inulin (M.W. 5000), and polyvinylpyrrolidone (average M.W. 33000 ). Thus the molecular weight range of the substances under study largely covered the spectrum of normal plasma solutes up to the size of plasma proteins.

\section{Methods}

ANIMALS AND TRACERS

Twenty-four female guinea-pigs weighing 500-750 g were used for this study. The substances whose compartmental distribution was to be investigated were ${ }^{14} \mathrm{C}$-urea, ${ }^{3} \mathrm{H}$-mannose, ${ }^{3} \mathrm{H}$-inulin, and ${ }^{125} \mathrm{I}$-polyvinylpyrrolidone (PVP) (Radiochemical Centre, Amersham).

The guinea-pigs were anaesthetised by ether inhalation followed by intraperitoneal pentobarbitone sodium (Nembutal) in a dose of $30 \mathrm{mg} / \mathrm{kg}$ body weight. With the animal supine the abdomen was opened by a short midline incision and a loop of jejunum was gently drawn out. One of the radioactive tracer substances was then administered to the animal by the intracardiac route in $2 \mathrm{ml}$ normal saline. The amounts of each substance given were as follows:

$\begin{array}{lr} & \mu c i \\ & 100 \\ { }^{14} \text { C-urea } & 100 \\ { }^{3} \text { H-mannose } & 200 \\ \text { 3H-inulin } & 1000 \\ \text { 125I-PVP } & 250\end{array}$

Biopsies $5 \mathrm{~mm}$ in length of the exteriorised loop were then taken at half, one, two, three, four, five, and 60 minutes after injection, ensuring that the area had a good blood supply at the time of biopsy. The biopsies were frozen immediately by immersion in isopentane cooled to $-155^{\circ} \mathrm{C}$ with liquid nitrogen. They were then temporarily stored in liquid nitrogen until sections were cut.

TISSUE PROCESSING AND AUTORADIOGRAPHY Coverslips had been previously prepared in the dark with a covering of glycerin albumin followed by stripping film on one side, emulsion face upwards, then dried and cooled to $-5^{\circ} \mathrm{C}$.

Sections of the trimmed biopsy specimens $5 \mu \mathrm{m}$ in thickness were cut on a cryostat at $-25^{\circ} \mathrm{C}$. The sections were picked up onto the emulsion of the prepared coverslips in the dark with red safelight. The autoradiographs were exposed for six weeks at $-25^{\circ} \mathrm{C}$ in light-tight boxes. After exposure the autoradiographs were fixed in 5\% acetic ethanol in the dark. They were developed for four minutes with D19 developer, washed for 30 seconds, fixed with Kodafix fixative for two minutes, washed again, and finally dried. After an interval of 24 hours to allow hardening, the sections were stained with haematoxylin and eosin. All stages of development and staining were carried out at $18^{\circ} \mathrm{C}$.

\section{GRAIN COUNTING}

Grain counts were made using a quadrantic grid with a magnification $\times 1000$. Each grid quadrant covered an area equal to about three epithelial cells. From the sections 50 quadrants were counted over each of the following compartments: the epithelium of the crypt, mid-villus and villus tip, the lamina propria of the villus core, and mucosal vascular lumina. The grain counts for each compartment were averaged and compared with vascular luminal activity. Background counts were subtracted from the tissue counts.

\section{Results}

${ }^{14}$ C-UREA

Figure 1 and the Table show than ${ }^{14} \mathrm{C}$-labelled urea was distributed evenly throughout the intestinal mucosa, the grain concentration being uniform over vascular lumen, lamina propria of the villus core, and epithelium. Epithelial grain counts over villus tip, mid-villus, and crypt did not differ significantly. There was no appreciable variation between autoradiographs taken from one to 60 minutes after injection.

Thus, ${ }^{14} \mathrm{C}$-urea permeates with great rapidity from the vascular lumen into all submucosal and mucosal compartments within one minute of intravenous injection. There appears to be little or no barrier to urea permeation as far as the epithelial cellpresumably because of the small molecular size of urea.

\section{${ }^{3}$ H-MANNOSE}

From Fig. 2 and the Table it may be seen that ${ }^{3}$ H-labelled mannose was widely distributed throughout the mucosa, and the grain density appeared to be equal in the epithelium and in the villus core. There was no significant difference in grain concentration between the epithelium of villus tip, mid-villus, or crypt. Within the villus core there was no gradient between the vascular lumina and the extravascular space. There was no qualitative difference between 


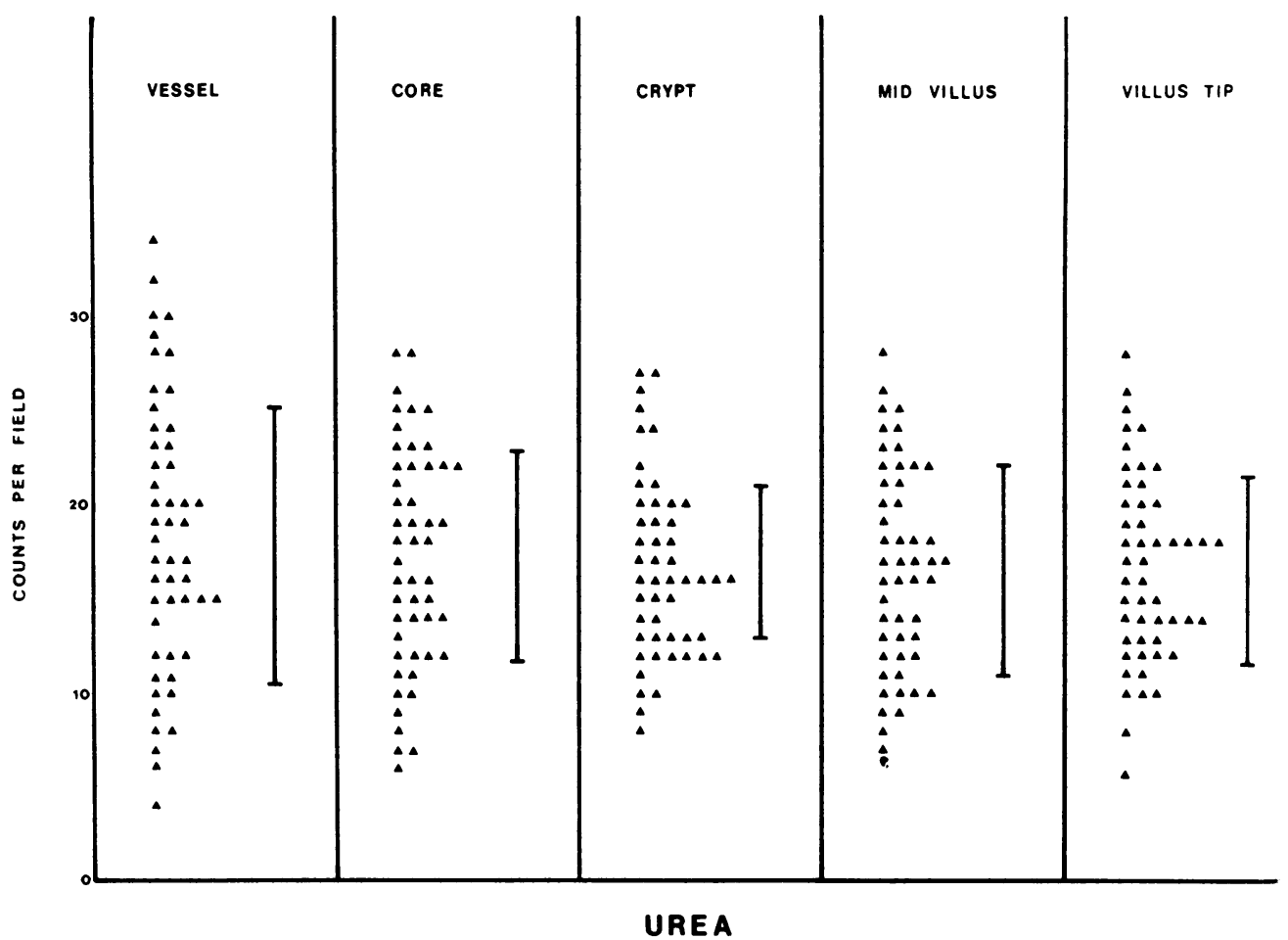

Fig. 1 The distribution of ${ }^{14} \mathrm{C}$ urea in epithelium (crypt, mid-villus and tip), villus core, and vascular lumen. Each point represents the grain count over one grid quadrant. The vertical bar indicates the one standard deviation range.

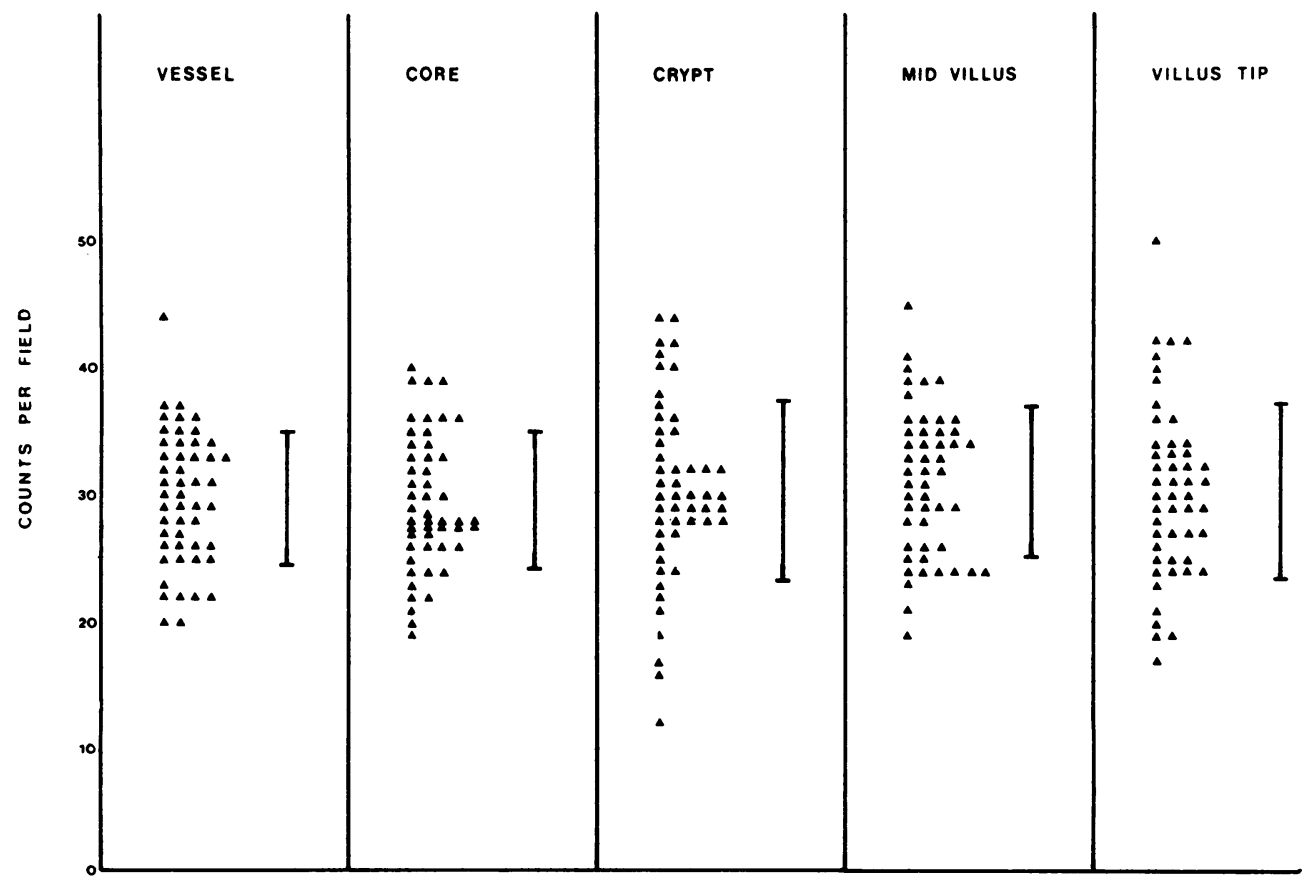

MANNOSE

Fig. 2 The distribution of ${ }^{3} \mathrm{H}$-mannose in epithelium (crypt, mid-villus and tip), villus core, and vascular lumen. Each point represents the grain count over one grid quadrant. The vertical bar indicates the one standard deviation range. 
Table Average grain counts $\pm S D$ of each of labelled tracer substances over crypt, mid-villus and tip epithelium, villus core and vascular lumen*

\begin{tabular}{|c|c|c|c|c|c|c|c|}
\hline Tracer substance & $M . W$. & & Vessel & Core & Crypt & Mid & Tip \\
\hline \multirow{2}{*}{ PVP } & \multirow{2}{*}{33000} & Average count & $312 \pm 74$ & $158 \pm 28$ & $21 \pm 4$ & $28 \pm 5$ & $43 \pm 7$ \\
\hline & & $\%$ of vessel count & 100 & 51 & 7 & 9 & 14 \\
\hline \multirow{2}{*}{ Inulin } & \multirow{2}{*}{5000} & Average count & $55 \pm 17$ & $52 \pm 15$ & $22 \pm 7$ & $19 \pm 6$ & $22 \pm 5$ \\
\hline & & $\%$ of vessel count & 100 & 95 & 40 & 36 & 40 \\
\hline \multirow{2}{*}{ Mannose } & \multirow{2}{*}{180} & Average count & $30 \pm 5$ & $30 \pm 5$ & $30 \pm 7$ & $31 \pm 6$ & $30 \pm 7$ \\
\hline & & $\%$ of vessel count & 100 & 100 & 100 & 103 & 100 \\
\hline \multirow{2}{*}{ Urea } & \multirow{2}{*}{60} & Average count & $18 \pm 7$ & $17 \pm 6$ & $17 \pm 4$ & $16 \pm 6$ & $17 \pm 5$ \\
\hline & & $\%$ of vessel count & 100 & 94 & 95 & 89 & 94 \\
\hline
\end{tabular}

*The compartmental counts are also expressed as a percentage of the counts over the vascular lumen.

autoradiographs taken at times varying from one to 60 minutes after ${ }^{3} \mathrm{H}$-mannose injection.

It is clear that ${ }^{3} \mathrm{H}$-mannose with a molecular weight of 180 appears to cross the barriers between vascular lumen and epithelium rapidly and freely. This was demonstrated by the equal grain concentrations in all compartments of the mucosa as early as one minute after injection. Thus the compartmental distribution of mannose is very similar to that of urea, which is not unexpected as they are both small molecules.

\section{${ }^{3}$ H-INULIN}

The distribution of ${ }^{3} \mathrm{H}$-labelled inulin is shown in Fig. 3 and the Table. The grain density over the epithelium was much less than over the villus core $(\mathrm{P}<0.001)$. There was no significant difference in grain counts between the epithelial cells of villus tip, mid-villus, or crypt. Within the villus core there was no significant gradient between the vascular lumina and the extravascular compartments. The concentration gradient between lamina propria and epithelium was approximately 5:2. Autoradiographs taken between one and 60 minutes after injection did not vary significantly.

Thus it is shown that ${ }^{3} \mathrm{H}$-inulin with a molecular weight of 5000 appears to cross the capillary wall rapidly and freely as judged by the equal grain concentrations between vascular lumen and extravascular space as early as one minute after injection. There is a concentration gradient of grain activity of approximately $5: 2$ between the lamina propria and the epithelium but there is no apparent difference between villus tip and crypt epithelial activity.

It is not possible to state definitely whether the grains counted over the epithelium originated from activity in the intercellular spaces or within the cells themselves. The grains over the epithelium appear to be uniform rather than in lines along the intercellular spaces, so it is probable that the grains originated from activity in both the intercellular and intracellular compartments.

The implication of the inulin results is that there is a barrier to permeation situated between the lamina propria and the epithelium; this may be either the epithelial basement membrane or the epithelial cell membrane.

\section{I-P VP}

Figure 4 and the Table show that ${ }^{125}$ I-labelled PVP exhibited the most notable differences in compartmental distribution. It may be seen that there was a high grain density over the vascular lumina, a moderate grain density over the extravascular villus core, but little activity over the epithelium. There appeared to be rather more activity over the epithelium of the villus tips than the crypts, with the activity gradually diminishing down the sides of the villi towards the crypt orifices. The differences between vessel, core, and epithelial counts were significant at the $1 \%$ level, as were the differences between crypt, mid-villus, and villus tip epithelial counts. The appearances of autoradiographs from biopsies taken one to 60 minutes after 125I-PVP injection were similar.

The Table shows that the concentration gradient of PVP between vascular lumen and extravascular villus core is approximately $2: 1$, whereas between core and epithelium it averages approximately $5: 1$. It is also apparent that the grain concentration decreased from villus tip down to crypt, the ratio between the two being 2:1, with the concentration over the mid-villus region falling between the two.

Thus polyvinylpyrrolidone (M.W. 8000-80 000, 


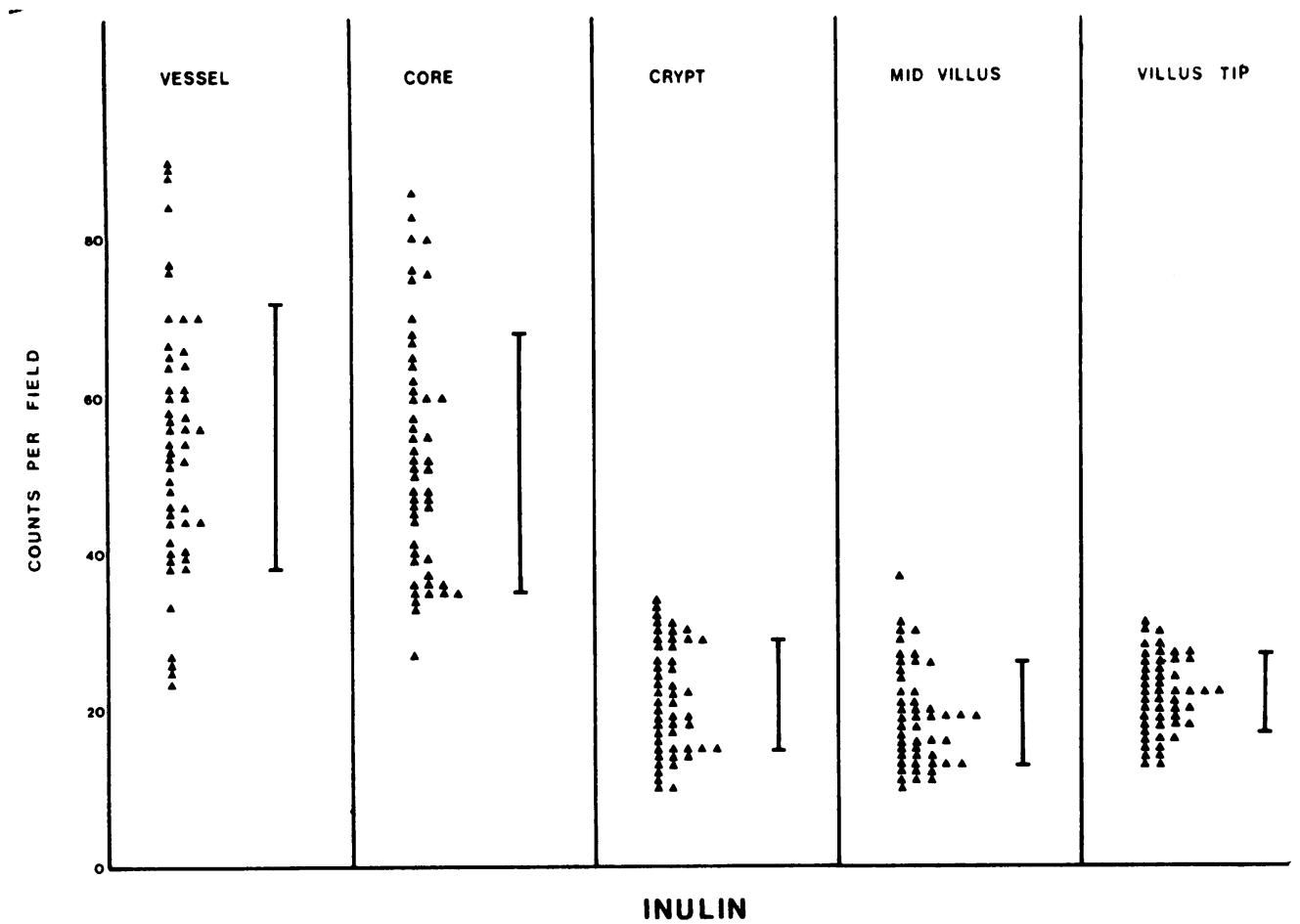

Fig. 3 The distribution of ${ }^{3} \mathrm{H}$-inulin in epithelium (crypt, mid-villus and tip), villus core, and vascular lumen. Each point represents the grain count over one grid quadrant. The vertical bar indicates the one standard deviation range.

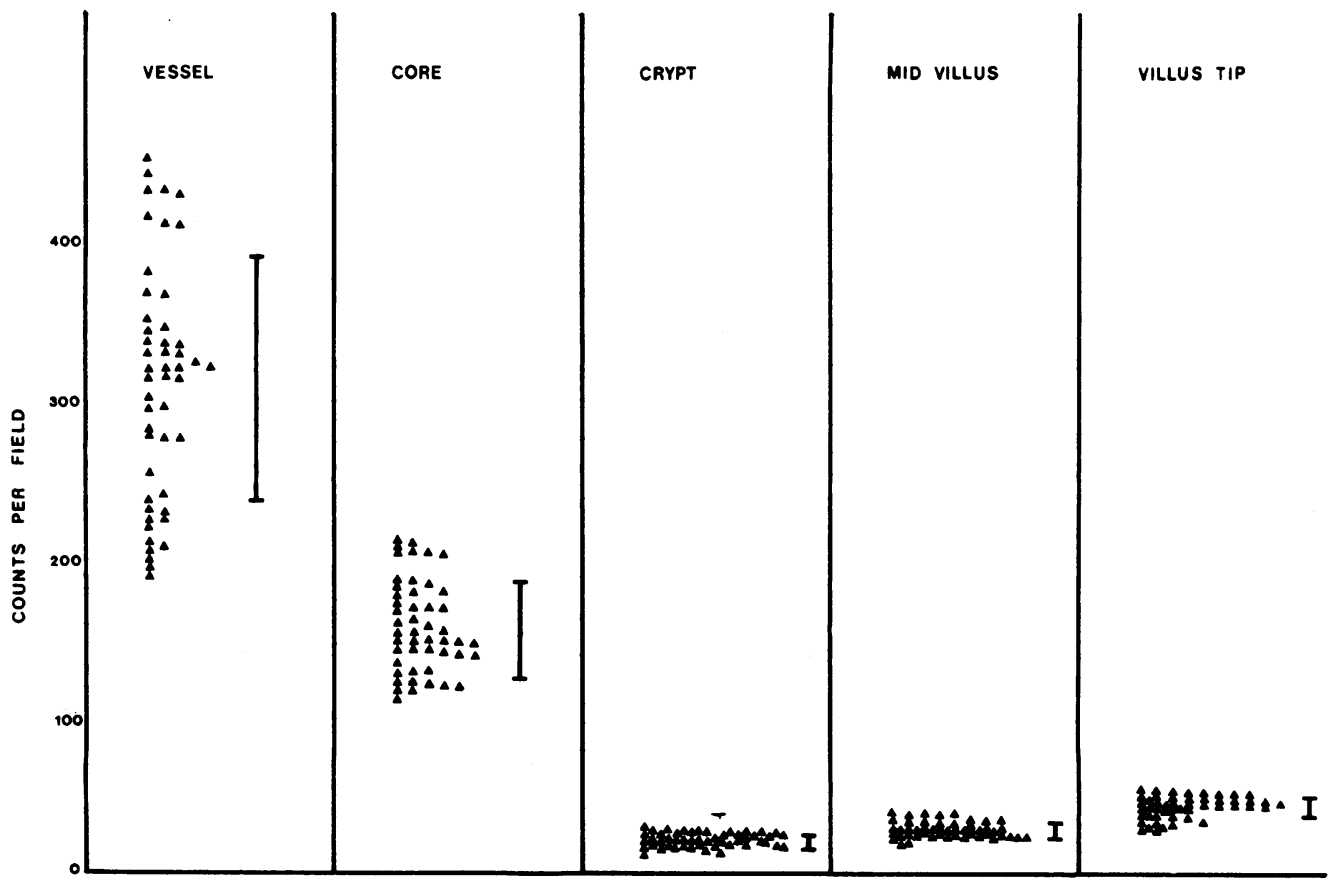

P. V.P.

Fig. 4 The distribution of ${ }^{125}$ I PVP in epithelium (crypt, mid-villus and tip), villus core, and vascular lumen. Each point represents the grain count over one grid quadrant. The vertical bar indicates the one standard deviation range. 
average M.W. 33000 ), with a molecular weight range similar to that of plasma proteins, appears in all compartments of the small intestinal wall and mucosa within one minute of intravenous injection. Therefore, despite its large size, this molecule permeates through the tissue at the same speed as urea, mannose, and inulin. However, there is obvious restriction of permeation, firstly, from the vascular lumen into the lamina propria, and, secondly, from the lamina propria into the epithelium. There is a certain degree of scatter in autoradiographic studies using ${ }^{125}$ I so that a small proportion of the grains counted in any compartment actually originated from activity in the adjacent compartment. In practice, this factitiously lessens the effect of the concentration gradients, especially when studying a single layer of cells such as intestinal epithelium. Therefore, the observed concentration gradient in fact underestimates the actual gradient. As with the inulin results, it is not possible to differentiate between inter- and intracellular activity over the epithelium and so the barrier effects of basement membrane and plasma membrane may not be separated.

It is interesting to speculate on the interpretation of the increased grain counts noted over the epithelium of the villus tips as compared with the more proximal epithelium. The cells of the villus tips are the oldest of the epithelial cells and are near the end of their life; at the extrusion zones themselves the cells are effete. The metabolic function of the cells diminishes as they migrate towards the extrusion zones, and, indeed, their morphological appearances change as well, with the cells of the villus tip showing minor degenerative changes of brush border irregularities and darkly staining nuclei. With these functional and morphological changes occurring in the senescent cells of the villus tips it seems not unlikely that the passive permeability of their plasma membranes may be altered. Certainly, cells which are damaged in any way tend to become more permeable. Thus it seems a reasonable assumption that the increased grain concentration noted over the epithelium of the villus tip is due to an increase in the passive permeability of the plasma membranes of these older cells.

\section{Discussion}

Previous autoradiographic studies in this field are few, although capillary permeability has been widely investigated using other techniques.

Clementi and Palade (1969a, b) used horseradish peroxidase (HRP) and ferritin as probe molecules for the small and large pore systems respectively in mouse intestinal capillaries. The substances were localised by direct light and electron microscopy after suitable staining. They found that HRP appeared in the pericapillary interstitial fluid within 75 seconds of intravenous injection and ferritin within three minutes. Equilibration took two and five minutes respectively. Passage across the endothelium was similar for both substances, being predominantly through the fenestrae and not apparently through the intercellular junctions. The concentration gradient across endothelium was slight for HRP but marked for ferritin. In neither case was there a concentration gradient across the endothelial basement membrane. If the endothelium were first made more permeable by histamine or EDTA and colloidal gold or carbon used as probes, then these particles came to rest on the endothelial basement membrane. Thus the basement membrane was shown to be freely permeable to HRP (molecular diameter $50 \AA$ ) and ferritin (M.D. $110 \AA$ ) but impermeable to colloidal gold (M.D. $220 \AA$ ) and carbon (M.D. $340 \AA$ ).

Our findings are in accordance with those of Clementi and Palade (1969a, b). From their results the inference may be drawn that the endothelial basement membrane should be freely permeable to all of the tracer molecules used in the present study, PVP having a molecular diameter similar to HRP.

Of the previous autoradiographic studies the most detailed is by Brooks and Dobbins (1972); it involved localisation of labelled albumin in guinea-pig intestine and the findings were essentially similar to ours. The highly purified autologous albumin was specially labelled with ${ }^{125}$ I and uncontaminated by free iodine. The highest grain density was seen over the vascular lumina, moderate density over the extracellular and epithelial intercellular spaces, and low density over the epithelial cells. The cells of the villous tips showed more ${ }^{125}$ I activity than the rest of the epithelium. The relative compartmental distribution varied little from one minute to two hours. At 30 minutes the ratio of grain concentration in vascular lumen, intercellular or extracellular space, and epithelial cell was approximately 40:10:1. Thus there were two major areas of restriction to albumin permeation: a 4:1 gradient between capillary lumen and extravascular space, and a 10:1 gradient between extravascular space and epithelial cell.

An earlier, but less comprehensive, autoradiographic study was made by Ullberg et al. (1960) to investigate the distribution of intravenously administered 131I albumin in cat and mouse. The animals were killed two to four hours after injection and autoradiograms made from $20 \mu \mathrm{m}$ sections of the whole fixed animals. Their results showed that the greatest accumulation of the radioactive label was in the gastric and small intestinal epithelium and lumen. They concluded that the intestinal radio- 
activity must have entered the bowel by excretion of 131I-albumin through the intestinal mucosa. However, from first principles it seems unlikely that after two to four hours the greatest concentration of intravenously administered albumin should be found in the intestinal lumen and epithelium, as this would imply that there was effectively little or no barrier to albumin passage between blood and gut lumen. Except in the most extreme cases of protein-losing enteropathy, this is known not to be the case. A possible explanation of their findings is that the fixing process split the iodine label from the albumin; the free ${ }^{131}$ Iodide could then, because of its small molecular size, diffuse unhampered into the intestinal lumen. In fact, their results may represent autoradiographs of the distribution of ${ }^{131}$ Iodide rather than 131I-labelled albumin.

Indeed, early autoradiographic studies in the present work involved formalin fixation of sections of small intestine after intravenous administration of ${ }^{125}$ I-labelled albumin. The resulting autoradiographs showed similar results to those of Ullberg et al. and seemed contradictory to existing knowledge of albumin passage into the intestine. In view of this, the experiments were repeated using 125I-PVP and a frozen autoradiographic technique to prevent diffusion. Using this technique the results were completely different and much more in keeping with predictions from the results of the other autoradiographic experiments in this study and those of other workers.

Thus our findings corroborate and complement those of Brooks and Dobbins (1972) and of Clementi and Palade (1969a, b). We have shown that the compartmental distribution within the small intestine varies with different solutes. Small solutes such as urea and mannose are distributed widely throughout all the interstitial and cellular compartments of the small intestinal mucosa and submucosa. Macromolecular solutes like PVP (M.W. 8000-80 000) have a very different distribution-the greatest concentration being within the vascular lumina with a lower concentration in the extravascular compartment of the submucosa. Within the epithelial cell layer there is a lower concentration still, although from the results of this study it was not possible to state whether the activity observed over the epithelium originated from intra- or extracellular PVP. There are, therefore, apparently two barriers to macromolecular permeation which are not barriers to small solutes (1) between vascular lumen and lamina propria - that is, the capillary wall-and (2) between lamina propria and epithelium - that is, either the epithelium basement membrane or the epithelial cell membrane.
The results of the inulin experiments fall between the two extreme pictures for very small (urea, mannose) and very large (PVP) solutes. There is no apparent barrier to permeation between vessel lumen and lamina propria, but there is a quite marked concentration gradient between the extravascular space and the epithelium. The implication from this finding is that the barrier on the epithelial side is more selective than that on the endothelial side.

The composite picture built up by the distribution of these four different sized solutes is of two definite selective barriers, one situated in the capillary wall and the other situated in the epithelium or its basement membrane. The selectivity of the barrier on the epithelial side is greater than that of the capillary barrier.

\section{References}

Barth, W. F., Wochner, R. D., Waldmann, T. A., and Fahey, J. L. (1964). Metabolism of human gamma macroglobulins. Journal of Clinical Investigation, 43, 1036-1048.

Bockman, D. E., and Winborn, W. B. (1966). Light and electron microscopy of intestinal ferritin absorption. Observations in sensitised and non-sensitised hamsters (Mesocricetus auratus). Anatomical Record, 155, 603-621.

Brooks, S. G., and Dobbins, W. O. (1972). Autoradiographic localisation of 1-125 labelled albumin in the intestine of guinea pigs: a light and electron microscopic study. Gastroenterology, 62, 1001-1012.

Clementi, F., and Palade, G. E. (1969a). Intestinal capillaries. I. Permeability to peroxidase and ferritin. Journal of Cell Biology, 41, 33-58.

Clementi, F., and Palade, G. E. (1969b). Intestinal capillaries. II. Structural effects of EDTA and histamine. Journal of Cell Biology, 42, 706-714.

Fordtran, J. S., Rector, F. C., Jr., Ewton, M. F., Sotor, N., and Kinney, J. (1965). Permeability characteristics of the human small intestine. Journal of Clinical Investigation, 44, 1935-1944.

Jarnum, S., and Jensen, K. B. (1972). Plasma protein turnover (albumin, transferrin, IgG, IgM) in Ménétrier's disease (giant hypertrophic gastritis): evidence of non-selective protein loss. Gut, 13, 128-137.

Kingham, J. G. C., and Loehry, C. A. (1976). Permeability of the small intestine after intra-arterial injection of histamine-type mediators and irradiation. Gut, 17, 517-526.

Kingham, J. G. C., Whorwell, P. J., and Loehry, C. A. (1976). Small intestinal permeability: 1 . Effects of ischaemia and exposure to acetyl salicylate. Gut, 17, 354-361.

Kraehenbuhl, J. P., and Campiche, M. E. (1969). Early stages of intestinal absorption of specific antibodies in the newborn. An ultrastructural, cytochemical, and immunological study in the pig, rat, and rabbit. Journal of Cell Biology, 42, 345-365.

Loehry, C. A., Axon, A. T. R., Hilton, P. J., Hider, R. C., and Creamer, B. (1970). Permeability of the small intestine to substances of different molecular weight. Gut, 11, 466-470.

Ullberg, S., Birke, G., Ewaldsson, B., Hansson, E., Liljedahl, S. O., Plantin, L. O., and Wetterfors, J. (1960). The role of the gastrointestinal tract in the elimination of serum albumin. A preliminary autoradiographic study. Acta Medica Scandinavica, 167, 421-425. 